\title{
BLIND SOURCE EXTRACTION OF PERIODIC SIGNALS
}

\author{
Foad Ghaderi, Hamid R. Mohseni, John G. McWhirter and Saeid Sanei \\ Centre of Digital Signal Processing, \\ School of Engineering, \\ Cardiff University, UK
}

\begin{abstract}
A new algorithm is developed here for blind extraction of periodic signals. It is assumed that the fundamental frequencies of the sources (or alternatively one of the harmonics for each source) are known a priori. Necessary and sufficient conditions for blind source extraction of cyclostationary signals are introduced and the optimization problem is solved using steepest descent method for complex matrices. Computer simulation results verify the effectiveness and good performance of the algorithm.
\end{abstract}

Index Terms - Blind source extraction, cyclostationarity, periodic signal separation.

\section{INTRODUCTION}

During the last decades much effort has been made by researchers for separating unknown mixed signals and estimating original source signals. In these problems the mixing mechanism is often unknown. In general, it is impossible to solve these problems unless some assumptions are made about the medium, nature, or number of the sources and measurements.

There are many processes in nature that originate from periodic phenomena. These processes may cause some random data that represent periodicity in their statistical properties, and are called cyclostationary processes. Examples of cyclostationary data can be found in telecommunication, radar and sonar applications, mechanics, econometrics, biological science and meteorology [1].

Higher order statistics are widely used in Blind Source Separation (BSS) problems and many algorithms have been developed based on this information up to now. A typical algorithm performs a pre-whitening operation followed by a rotation to find the estimations.

In [2] the periodicity of the correlation matrix of cyclostationary signals is used to separate periodic signals. In this attempt, the covariance matrices have been diagonalized in different lags. The second-order blind identification (SOBI) algorithm is another well known method in which the covariance matrices with different lags are jointly diagonalized [3].
Convolutive mixtures of cyclostationary signals have been considered as well. In [4] the conventional higher-order statistics and second-order cyclostationarity of the signals are used and it is shown that exploitation of the statistical properties of this kind of signals can enhance the performance of blind cyclostationary source separation.

In this paper, it is assumed that the source signals are second order cyclostationary. The necessary and sufficient conditions for signals to be blindly separated are introduced and an algorithm based on steepest descent method for complex matrices on differential manifolds is developed for diagonalizing the cyclic correlation matrix under unitary constraint.

Definitions of the concepts and problem formulation are presented in the next section. In section 3 the proposed algorithm is explained. Experimental results are mentioned in section 4 and the concluding remarks are given in the last section.

\section{PROBLEM FORMULATION}

Assume $m$ unit norm mutually statistically independent unknown sources are mixed instantaneously through an unknown medium and $n$ sensors $(n \geq m)$ are used to measure these signals, as in Fig. 1. This system can be formulated in vector form as

$$
\mathbf{x}(t)=\mathbf{A s}(t)+\mathbf{n}(t)
$$

where $\mathbf{s}(t)=\left[s_{1}(t) \ldots s_{m}(t)\right]^{T}$ is the $m \times 1$ source vector, $\mathbf{n}(t)=\left[n_{1}(t) \ldots n_{n}(t)\right]^{T}$ is an $n \times 1$ stationary noise vector, $\mathbf{x}(t)=\left[x_{1}(t) \ldots x_{n}(t)\right]^{T}$ is the $n \times 1$ measurement vector, $\mathbf{A}$ is an $n \times m$ unknown full column rank mixing matrix and superscript $T$ represents the transpose of a vector. It is assumed that the source signals are periodic with distinct fundamental frequencies, thus they are second order cyclostationary in wide sense [1]. Furthermore, to simplify the problem we assume that $m=n$.

To estimate the original sources, the observations are first pre-whitened to obtain $\mathbf{z}(t)=\mathbf{W} \mathbf{x}(t)=\mathbf{C s}(t)$, where $\mathbf{C}=$ WA (see Fig. 1). To find an estimation of the source signals we need to rotate the whitened data by a matrix $\mathbf{B}$, which will generate $\hat{\mathbf{z}}(t)=\mathbf{B}^{H} \mathbf{z}(t)$. In [5], fourth order cumulants are used to find the rotation matrix for non-Gaussian sources. 


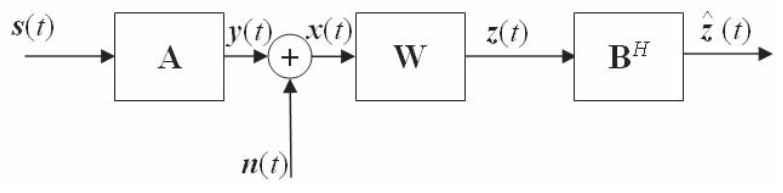

Fig. 1. The overall block diagram.

Here we exploit the cyclostationarity properties of the sources to estimate and extract the original sources.

It is shown in [6] that if $s(t)$ is cyclostationary, then this process and its frequency-shifted version $s(t) e^{j 2 \pi \beta t}$ are correlated for some $\beta$, where $\beta$ s are called cyclic frequencies of $s(t)$. This property results in the definition of cyclic correlation of the signals. The cyclic cross-correlation function of the elements $x_{p}(t)$ and $x_{q}(t)$ of the complex vector $\mathbf{x}(t)$ at cycle frequency $\beta$ and lag $\tau$ is defined as

$$
r_{p q}^{\beta}(\mathbf{x}, \tau)=\left\langle x_{p}(t) x_{q}^{\star}(t+\tau) e^{-j 2 \pi \beta t}\right\rangle
$$

where $\langle$.$\rangle denotes time averaging operator, i.e. \lim _{T \rightarrow \infty} \frac{1}{2 T+1}$ $\sum_{t=-T}^{T} x_{p}(t) x_{q}^{\star}(t+\tau) e^{-j 2 \pi \beta t},(.)^{\star}$ represents complex conjugate of a complex variable, and $j=\sqrt{-1}$. Accordingly, cyclic correlation matrix of the $n \times 1$ complex signal vector $\mathbf{x}(t)$ is defined as:

$$
\mathbf{R}_{\mathbf{x}}^{\beta}(\tau) \triangleq\left\langle\mathbf{x}(t) \mathbf{x}^{H}(t+\tau) e^{j \beta t}\right\rangle
$$

where superscript $H$ denotes complex conjugate transpose of a matrix. If $\mathbf{s}(t)$ is a vector of $n$ independent signals with distinct cycle frequencies, cyclic cross-correlation function has the following properties [7]:

$$
\begin{array}{ll}
r_{p q}^{\beta_{p}}(\mathbf{s}, \tau)=\left\langle s_{p}(t) s_{q}^{\star}(t+\tau) e^{j \beta_{p} t}\right\rangle=0, & \text { if } p \neq q \\
r_{p p}^{\beta_{q}}(\mathbf{s}, \tau)=\left\langle s_{p}(t) s_{p}^{\star}(t+\tau) e^{j \beta_{q} t}\right\rangle=0, & \text { if } p \neq q \\
r_{p p}^{\beta_{p}}(\mathbf{s}, 0)=\left\langle s_{p}(t) s_{p}^{\star}(t) e^{j \beta_{p} t}\right\rangle \neq 0, & \forall p
\end{array}
$$

where $\beta_{i}$ is the cycle frequency of the source $s_{i}(t)$. This property as will be seen in this paper can be used for blindly extracting almost cyclostationary stochastic processes. The following theorem considers the necessary and sufficient conditions for extracting one cyclostationary source from the set of observations.

Theorem 1. Assume $\boldsymbol{z}(t)$ is a white mixture of cyclostationary sources with distinct cycle frequencies $\beta_{i}, i \in\{1, \ldots, n\}$. For any unitary matrix $\boldsymbol{B}$, define $\hat{\boldsymbol{z}}(t)=\boldsymbol{B}^{H} \boldsymbol{z}(t)$. Then $\boldsymbol{b}_{i}$ (column $\boldsymbol{i}$ of $\boldsymbol{B})$ is an extracting vector of pth source if and only if $\left|r_{i i}^{\beta_{p}}(\hat{\mathbf{z}}, 0)\right|=1$ and $r_{i j}^{\beta_{p}}(\hat{\mathbf{z}}, 0)=0$ for all $j \in\{1, \ldots, i-1, i+$ $1, \ldots, n\}$

This theorem can be proved easily by expanding $r_{i j}^{\beta_{p}}(\hat{z}, 0)$ with respect to the elements of $\mathbf{R}_{\mathbf{s}}^{\beta_{p}}(0)$ and the elements of the matrix $\mathbf{D}=\mathbf{B}^{H} \mathbf{C}$.
Therefore, if a matrix $\mathbf{B}$ can be found such that the conditions of theorem 1 are satisfied, the mixed sources can be extracted one by one. We now return to the problem of extracting periodic sources. To simplify the calculations, it is assumed that the sources are real, and it is easy to extend the results to complex case. Fourier series expansion of the periodic sources exist and for $v$ th source it can be written as:

$$
s_{v}(t)=\alpha_{v 0}+\sum_{l=1}^{L} \alpha_{v l} \cos \left(2 \pi l f_{v} t+\phi_{l}\right)
$$

where $f_{v}$ is the fundamental frequency of the source $v$, and $L$ is the number of coefficients in the Fourier series expansion. Since it is assumed that the source signals are zero mean, then $\alpha_{v 0}=0$. To calculate $r_{p q}^{\beta}(\hat{\mathbf{z}}, 0)$ we first evaluate $\hat{z}_{p}(t) \hat{z}_{q}(t)$ for all $p$ and $q$ as

$$
\begin{aligned}
\hat{z}_{p}(t) \hat{z}_{q}(t)= & \sum_{h=1}^{n} d_{p h} s_{h}(t) \sum_{g=1}^{n} d_{q g} s_{g}(t) \\
= & \sum_{h=1}^{n} \sum_{g=1}^{n} d_{p h} d_{q g} \sum_{l=1}^{L} \sum_{e=1}^{E} \alpha_{h l} \alpha_{g e} \\
& \times \cos \left(2 \pi l f_{h} t+\phi_{l}\right) \cos \left(2 \pi e f_{g} t+\phi_{e}\right)
\end{aligned}
$$

From basic trigonometry we know that

$$
\begin{gathered}
\cos \left(2 \pi l f_{h} t+\phi_{l}\right) \cos \left(2 \pi e f_{g} t+\phi_{e}\right)= \\
\frac{1}{2}\left[\cos \left(2 \pi\left(l f_{h}+e f_{g}\right) t+\phi_{l}+\phi_{e}\right)+\right. \\
\left.\cos \left(2 \pi\left(l f_{h}-e f_{g}\right) t+\phi_{l}-\phi_{e}\right)\right]
\end{gathered}
$$

Substituting (6) and (7) in (2) and remembering the Fourier transform properties of cosine function and ignoring the frequency component at $\beta=0$ it can be concluded that:

- For a source signal of the form (5), $r_{v v}^{\beta}(\mathbf{s}, 0)$ can have nonzero values for all $\beta_{v} \in \mathcal{F}_{v}$, where $\mathcal{F}_{v}=\{\beta \mid \beta=$ $\left.k f_{v} ; \forall k \in\{1, . ., 2 L\}, \beta \neq 0\right\} . \beta_{v}$ is a cyclic frequency of source $v$.

- The elements of $\mathbf{R}_{\hat{\mathbf{z}}}^{\beta}(0)$ can have nonzero values at cyclic frequencies $\beta$, where $\beta \in\left\{l f_{1} \pm e f_{1}, \ldots, l f_{1} \pm\right.$ $\left.e f_{n}, \ldots, l f_{n} \pm e f_{1}, \ldots, l f_{n} \pm e f_{n}, \beta \neq 0\right\}$

In other words, the spectrum of the quadratic function $\hat{z}_{p}(t) \hat{z}_{q}(t)$ has a nonzero component at some $\beta$, where the value of this component is composed of two parts. The first one is resulted from a cosine term with frequency $f=\beta / 2$ and the second one comes from the values of cross components which are created by mutually multiplying the harmonics of the source signals while their harmonic frequencies satisfy:

$$
l f_{h} \pm e f_{g}=\beta
$$

For the $i$ th source with cycle frequency $\beta_{i}$, provided that the source signals satisfy

$$
\forall h, g \neq i \quad l f_{h} \pm e f_{g} \neq \beta_{i}
$$


if a matrix $\mathrm{B}$ is found such that for $\beta_{i} \in \mathcal{F}_{i}$

$$
\text { off }\left\{\mathbf{R}_{\hat{\mathbf{z}}}^{\beta_{i}}(0)\right\}=0
$$

it means that the cosine term with the frequency of $f=\beta_{i} / 2$ exists in just one row of $\widehat{\mathbf{z}}(t)$. Operator off $\{$.$\} computes the$ sum of the squared magnitudes of the off-diagonal elements of a matrix. Since we assume that the sources are unit norm, this cosine term is the dominant component of that element. In other words, $\mathbf{B}$ can extract a cosine term with frequency $\beta_{i}$ in the source of interest. It is shown in [8] that diagonalization of $\mathbf{R}_{\hat{\mathbf{z}}}^{\beta_{i}}(0)$ means that $\mathbf{D}$ is a unitary diagonal matrix, thus, when extracting the cosine term all the harmonics of the $i$ th source are extracted by $\mathbf{B}$.

This means that to extract the $i$ th sourcre we can relax the condition of theorem 1 and simply diagonalize the cyclic correlation matrix of estimation vector.

Although (9) imposes a restriction, it provides some redundancy in selecting the appropriate cycle frequency. In other words, we don't need to select the fundamental cycle frequency (the one corresponding to the fundamental frequency) as the required cycle frequency of the source. As long as the cycle frequencies satisfy (9), there is no more restriction in selecting them.

\section{ALGORITHM}

Our objective is to find a matrix $\mathbf{B}$ for which (10) is satisfied. Thus the following cost function is defined, which is a measure of nondiagonality of $\mathbf{R}_{\hat{\mathbf{Z}}}^{\beta_{p}}(0)$ :

$$
\mathcal{J}(\mathbf{B})=\operatorname{off}\left\{\mathbf{R}_{\hat{\mathbf{z}}}^{\beta_{p}}(0)\right\}=\operatorname{off}\left\{\mathbf{B}^{H} \mathbf{R}_{\mathrm{z}}^{\beta p}(0) \mathbf{B}\right\}
$$

In order to find the minimum point of $\mathcal{J}(\mathbf{B})$ we use the steepest descent method for complex matrices [9]. This method stems from differential geometry and optimizes the cost function under unitary constraint. It is shown in [9] that to find the optimum point of a cost function under unitary constraint on the Riemannian space it is enough to evaluate the gradient of the cost function at a point $\mathbf{B}$ and then translate it to identity. In mathematical terms, if $\nabla \mathcal{J}(\mathbf{B})$ is the gradient of the cost function $\mathcal{J}(\mathbf{B})$ on Euclidean space, then the gradient direction on the Riemannian space is:

$$
\widehat{\nabla}(\mathbf{B})=\nabla \mathcal{J}(\mathbf{B}) \mathbf{B}^{H}-\mathbf{B} \nabla \mathcal{J}(\mathbf{B})^{H}
$$

For our problem, the Euclidean gradient of $G(\mathbf{B})$ is

$$
\begin{aligned}
& \nabla \mathcal{J}(\mathbf{B})=\frac{\partial \mathcal{J}(\mathbf{B})}{\partial \mathbf{B}^{\star}} \\
& =\mathbf{R}_{\mathrm{z}}^{\beta p}(0) \mathbf{B}\left(\mathbf{B}^{H} \mathbf{R}_{\mathrm{z}}^{\beta p}(0) \mathbf{B}-\operatorname{diag}\left(\mathbf{B}^{H} \mathbf{R}_{\mathrm{z}}^{\beta p}(0) \mathbf{B}\right)\right)
\end{aligned}
$$

where $\operatorname{diag}($.$) denotes diagonal elements of a matrix [10].$

The Riemannian steepest descent algorithm [9] is presented in procedure PSE.

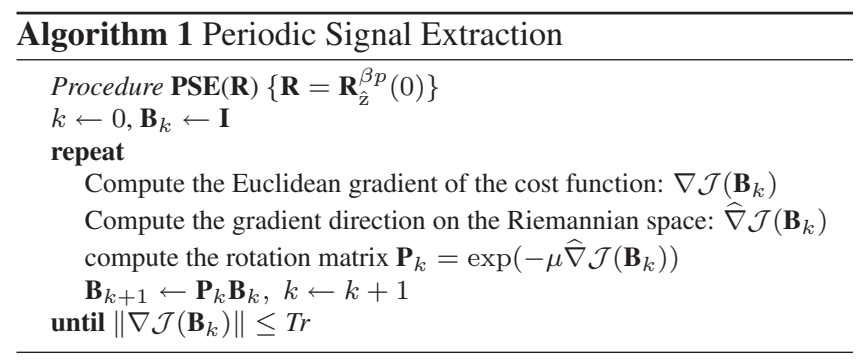

where $\mu$ is the learning rate and the exponential of an $n \times n$ complex matrix is given by the convergent power series $\exp (\mathbf{A})=\sum_{m=0}^{\infty}\left(\mathbf{A}^{m} / m !\right)$.

After convergence of the algorithm, the $p$ th source will appear in one of the rows of $\hat{\mathbf{z}}(t)$, where due to the permutation ambiguity the row number is not known. At this stage we have to deflate the extracted signal from the mixtures. The conventional batch deflation methods (see [11]) use the standard second order statistics as a measure of uncorrelatedness. Therefore there in no guarantee that the cyclic components of the extracted signal are well deflated. We define the following cost function:

$$
\begin{aligned}
\tilde{\mathcal{J}}_{p}\left(\tilde{\mathbf{b}}_{p}\right)= & \left\langle\mathbf{z}_{p+1}^{H}(t) \mathbf{z}_{p+1}(t) e^{j \beta_{p} t}\right\rangle \\
= & \left\langle\mathbf{z}_{p}^{H}(t) \mathbf{z}_{p}(t) e^{j \beta_{p} t}\right\rangle-2 \tilde{\mathbf{b}}_{p}^{H}\left\langle\mathbf{z}_{p}^{H}(t) \hat{z}_{p}(t) e^{j \beta_{p} t}\right\rangle \\
& +\tilde{\mathbf{b}}_{p}^{H} \tilde{\mathbf{b}}_{p}\left\langle\hat{z}_{p}^{2}(t) e^{j \beta_{p} t}\right\rangle
\end{aligned}
$$

where $\mathbf{z}_{1}(t)=\mathbf{z}(t), \hat{z}_{p}(t)$ is the $p$ th extracted source, $\mathbf{z}_{p+1}(t)=\mathbf{z}_{p}(t)-\tilde{\mathbf{b}}_{p} \hat{z}_{p}(t)$ and $\tilde{\mathbf{b}}_{p}$ is the $n \times 1$ deflating column vector. By differentiating $\widetilde{\mathcal{J}}_{p}\left(\tilde{\mathbf{b}}_{p}\right)$ with respect to $\tilde{\mathbf{b}}_{p}$ and solving the result to find the minimum point, we will have the following deflating equation:

$$
\tilde{\mathbf{b}}_{p}=\frac{\left\langle\mathbf{z}_{p}(t) \mathbf{z}_{p}^{H}(t) e^{j \beta_{p} t}\right\rangle \mathbf{b}_{p}}{\left\langle\hat{z}_{p}^{2}(t) e^{j \beta_{p} t}\right\rangle}
$$

where $\mathbf{b}_{p}$ is a column in $\mathbf{B}$ corresponding to source $p$.

\section{EXPERIMENTS}

Computer simulations were carried out to illustrate the performance of the proposed algorithm and the results were compared with those of SOBI, [3]. The objective of the proposed algorithm is to estimate the source signals such that they are uncorrelated and there is minimum cyclic correlation between them. So, the following performance index is used to measure the cyclic correlation between the estimated sources:

$$
P I=\sum_{i=1}^{m} \operatorname{off}\left\{\mathbf{R}_{\hat{\mathrm{z}}}^{\beta_{i}}(0)\right\}
$$

The proposed algorithm was applied to 2000 samples of three periodic signals (Fig. 2). Different levels of noise were added 

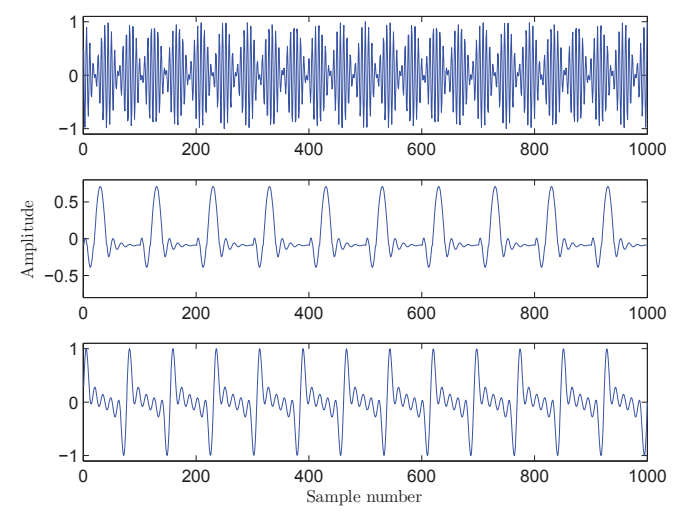

Fig. 2. Source signals

and the performance index was averaged over 100 independent trials for each level of noise. The signal-to-noise ratio is defined as SNR $=-10 \log _{10} \sigma^{2}$, where $\sigma^{2}$ is the noise variance.

The values of the performance indices for SOBI and PSE are presented in Fig. 3. As it can be seen, SOBI performs better just for high values of noise, while for normal noise levels, PSE provides a better performance.

\section{CONCLUDING REMARKS}

In this paper an algorithm for cyclostationary blind source extraction is introduced. The algorithm is based on diagonalization of the cyclic correlation matrix at a specific cycle frequency. Although the relationship between the cycle frequencies (and their harmonics) must comply with some conditions but practically such conditions are often satisfied. In places where the harmonics of the cycle frequencies exist, the conditions in (9) allows to select a different cycle frequency. Computer simulations show the performance of the algorithm.

\section{REFERENCES}

[1] W. A. Gardner, A. Napolitano, and L. Paura, "Cyclostationarity: half a century of research," Signal Process., vol. 86, no. 4, pp. 639-697, 2006.

[2] M. G. Jafari, W. Wang, J. A. Chambers, T. Hoya, and A. Cichocki, "Sequential blind source separation based exclusively on second-order statistics developed for a class of periodic signals.," IEEE Transactions on Signal Processing, vol. 54, no. 3, pp. 1028-1040, 2006.

[3] A. Belouchrani, K. Abed-Meraim, J.-F. Cardoso, and E. Moulines, "A blind source separation technique using second-order statistics," Signal Processing, IEEE Transactions on, vol. 45, no. 2, pp. 434-444, Feb. 1997.

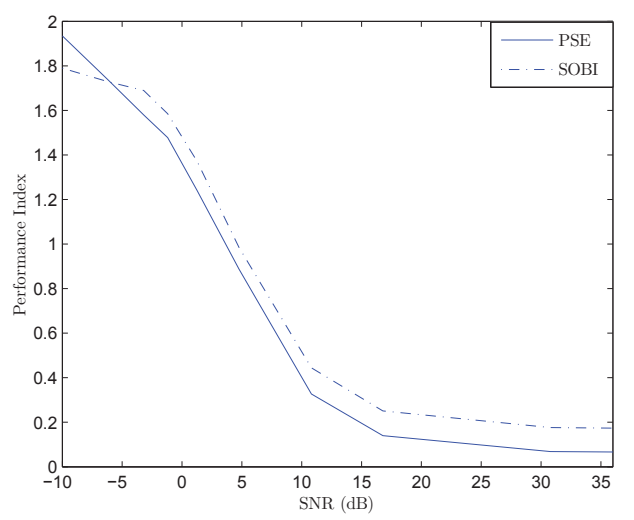

Fig. 3. Comparison of the performance of the proposed algorithm and SOBI for different levels of noise.

[4] W. Wang, M. G. Jafari, S. Sanei, and J. A. Chambers, "Blind separation of convolutive mixtures of cyclostationary sources using an extended natural gradient method," Signal Processing and Its Applications, 2003. Proceedings. Seventh International Symposium on, vol. 2, pp. 93-96 vol.2, July 2003.

[5] J. F. Cardoso and A. Souloumiac, "Blind beamforming for non-gaussian signals," Radar and Signal Processing, IEE Proceedings F, vol. 140, no. 6, pp. 362-370, Dec. 1993.

[6] W. A. Gardner, "Exploitation of spectral redundancy in cyclostationary signals," Signal Processing Magazine, IEEE, vol. 8, no. 2, pp. 14-36, Apr. 1991.

[7] K. Abed-Meraim, Yong Xiang, J. H. Manton, and Yingbo Hua, "Blind source-separation using secondorder cyclostationary statistics," Signal Processing, IEEE Transactions on, vol. 49, no. 4, pp. 694-701, Apr. 2001.

[8] F. Ghaderi, S. Sanei, and M. G. Jafari, "A unitary diagonalization method for blind cyclostationary source separation," ICA Research Network International Workshop, Sep. 2008.

[9] T. E. Abrudan, J. Eriksson, and V. Koivunen, "Steepest descent algorithms for optimization under unitary matrix constraint," Signal Processing, IEEE Transactions on, vol. 56, no. 3, pp. 1134-1147, March 2008.

[10] D. H. Brandwood, "A complex gradient operator and its application in adaptive array theory," IEE Proceedings F: Communications Radar and Signal Processing, vol. 130, pp. 11-16, feb. 1983.

[11] S. Amari and A. Cichocki, "Adaptive blind signal processing-neural network approaches," Proceedings of the IEEE, vol. 86, no. 10, pp. 2026-2048, Oct. 1998. 